\title{
Abstract: The Open Graph Protocol Design Decisions
}

\author{
Austin Haugen \\ Facebook, US
}

The Open Graph protocol enables any web page to become a rich object in a social graph. It was created by Facebook but designed to be generally useful to anyone. While many different technologies and schemas exist and could be combined together, there is not a single technology which provides enough information to richly represent any web page within the social graph. The Open Graph protocol builds on these existing technologies and gives developers one thing to implement. Developer simplicity is a key goal of the Open Graph protocol which has informed many of the technical design decisions. This talk will explore the motivation of the Open Graph protocol and the design decisions which went into creating it. 